ARTIGO ORIGINAL

\title{
INFLUÊNCIA DO TREINAMENTO CONCORRENTE DE CAPOEIRA E ATLETISMO NO DESEMPENHO MOTOR DE CRIANÇAS PRATICANTES DE FUTSAL
}

\author{
Influence of training and competing in athletics in capoeira \\ performance engine practitioners of children futsal
}

\author{
Evaldo Maciel Pinto ${ }^{1,2}$, Diogo Guimarães ${ }^{3}$, Athos Henrique Rocha Villaça4, Sebastião Lobo da Silva', Bibiano Madrid,6. \\ 1-Faculdade Albert Einstein (FALBE) - Brasília - Distrito Federal - Brasil. 2 - S Ten do Exército - C Doc EX - Brasília - Distrito Federal - Brasil. \\ 3 - Universidade Paulista (UNIP) - Brasília - Distrito Federal - Brasil. 4 - Centro Universitário de Brasília (UniCEUB) - Brasília - Distrito Federal - Brasil. \\ 5 - Laboratório de Avaliação Física e Treinamento (LAFIT) - Taguatinga - Distrito Federal - Brasil. 6 - \\ Programa de Pós-Graduação em Educação Física Universidade Católica de Brasília (UCB) - Taguatinga - Distrito Federal - Brasil.
}

\begin{abstract}
Palavras-chave: Treinamento. Futsal. Capoeira. Atletismo.
Influence of training and competing in athletics in capoeira performance engine practitioners of children futsal

Abstract: This study aimed to determine the influence of concurrent training and athletic capoeira levels of motor performance in children aged 7 to 10 years indoor football. Study participants were children $(n=39)$ were male. The subjects were divided into two groups, submitted to concurrent training, the AF group $(n=20)$ underwent the training of soccer and athletics and FC group $(n=19)$ soccer and capoeira. To study and evaluate the level of motor performance of the two sample groups, we applied a battery of tests for statistical analysis and checked the normality of the data (Kolmogorov-Smirnov) and applied repeated measures ANOVA with post hoc Bonferroni. We used the statistical packages SPSS (12.0) and Instat (3.0). The results note that in terms of motor proficiency children who practiced FC compared to children who practiced FA had equal values in the components of motor skills, however, both groups showed a trend in weight gain in their motor skills.
\end{abstract}

Keywords: Training. Futsal. Capoeira. Athletics.

Aceito em: 25/02/2011 - Revista de Educação Física 2012 Ago; 155: 9-13. Rio de Janeiro - Brasil

\section{INTRODUÇÃO}

O futsal é a modalidade esportiva mais praticada no Brasil, abrangendo todas as faixas etárias ${ }^{(1)}$. Este esporte vem constantemente passando por transformações tanto em suas regras como na forma de aprendizagem, tornando-se cada vez mais atraente e competitivo nos aspectos cognitivos, psicológicos, motores e para resolução de problemas mais complexos ${ }^{(2)}$.

O futsal é um esporte de movimentos velozes e reações rápidas, onde os jogadores têm que perceber estímulos, interpretá-los e programar respostas, tudo isso em um curto espaço de tempo com movimentos de elevado grau de coordenação(3).

Para a aprendizagem de um gesto motor, a criança que possuir mais experiências anteriores terá um nível mais elevado de desenvolvimento em relação à outra criança. Toda criança deve possuir uma diversidade de experiências, adquirindo assim, um desenvolvimento motor necessário para execução de tarefas mais complexas ${ }^{(4)}$. A variedade de experiências práticas aumenta a capacidade das pessoas de desenvolvê-las com sucesso em situações futuras ${ }^{(5)}$.

O atletismo é introduzido indiretamente em outras modalidades, seja através de elementos motores ou habilidades básicas com suas variantes( ${ }^{(6)}$. A iniciação ao atletismo é uma modalidade acessível à iniciação esportiva e irá aumentar o repertório motor da criança. Pode-se considerar que o atletismo é a base para as demais modalidades, visto que correr, saltar e lançar, estão presentes nas demais modalidades esportivas ${ }^{(7)}$.

$\mathrm{Na}$ capoeira, as qualidades físicas como coordenação, equilíbrio, velocidade, destreza, agilidade, flexibilidade e a resistência são trabalhadas e desenvolvidas em permanente movimentação(8). A capoeira como ferramenta pedagógica é recomendada pelos parâmetros curriculares nacionais (PCNs), sendo que a criança deve ser considerada como um todo, nos aspectos cognitivos, afetivos e corporais, construindo conhecimentos relativos ao corpo e ao movimento(9).

Os métodos de treinamento são todos os meios que propiciem o desenvolvimento das capacidades de movimentos e desenvolvam também, o máximo possível as capacidades motoras como força, velocidade, 
resistência, habilidade e flexibilidade ${ }^{(10)}$.

Segundo Leveritti apud Panissa(11), treinamento concorrente é a realização de exercícios de força e aeróbio na mesma sessão de treinamento. Existem outros tipos de treinamento combinado como o forçaflexibilidade por exemplo(12). Para Paulo(13), o rendimento esportivo pode ser afetado com a aplicação de protocolos de treinamento concorrente. Poucas pesquisas e estudos científicos têm sido realizados no que diz respeito à influência do treinamento concorrente no desempenho motor. Portanto, este estudo teve como objetivo verificar a influência do treinamento concorrente de atletismo e capoeira nos níveis de desempenho motor, em crianças de 7 a 10 anos praticantes de futsal.

\section{METODOLOGIA}

A amostra do presente estudo foi composta por crianças do sexo masculino, com idades entre 7 a 10 anos, participantes de escolinhas de futsal no mínimo há seis meses $(n=39)$. Os indivíduos foram divididos em dois grupos, submetidos ao treinamento concorrente, o grupo FA foi submetido ao treinamento de futsal $\mathrm{e}$ atletismo $(n=20)$ e o grupo FC a futsal e capoeira $(n=19)$.

$\mathrm{Na}$ realização do primeiro teste para o re-teste houve um intervalo de dois meses onde os avaliados do grupo FC, além das aulas normais de futsal, receberam a iniciação à capoeira, uma vez por semana como aula complementar. No mesmo intervalo os avaliados do grupo FA, além das aulas normais de futsal, receberam a iniciação do atletismo, como aula complementar.

Os alunos foram informados dos objetivos e da forma de execução das atividades por meio de uma demonstração, e foram motivados a apresentarem o seu melhor rendimento. Os voluntários, bem como seus pais, assinaram o termo de consentimento livre e esclarecido. Foi medida a massa corporal (Plenna precisão de 100 gramas) e estatura (Starrett precisão de 0,1 mm).

Para avaliação do desempenho motor, as crianças foram submetidas a uma bateria constituída de nove testes, pré e pós-treinamento. A bateria de testes foi executada para mensurar as seguintes variáveis: resistência abdominal, potência dos membros inferiores, velocidade de deslocamento, agilidade, precisão membros inferiores (chute a gol com bola parada e chute a gol com bola em movimento) e precisão do passe.

Para a avaliação da resistência abdominal, o avaliado assumiu a posição de decúbito dorsal, joelhos fletidos formando um ângulo de 90 graus, as plantas dos pés em pleno contato com o solo e os pés afastados a uma distância inferior a 30 centímetros, com os braços cruzados à frente do tronco, de forma que a mão direita toque o ombro esquerdo e a mão esquerda toque o ombro direito e a cabeça em contato com o solo. Um auxiliar permaneceu segurando o tornozelo do avaliado, para que o mesmo mantivesse um ângulo de 90 graus. $\mathrm{O}$ avaliado elevou o tronco até a posição sentado $\left(45^{\circ}\right)$ e retornou a posição inicial. A contagem foi realizada a partir do número de vezes que o aluno voltou à posição inicial, de maneira correta, em 1 minuto(14).

Para a avaliação da potência dos membros inferiores foi realizado um teste de impulsão horizontal, com o aluno partindo da posição em pé, pés paralelos e em pequeno afastamento lateral, com a trena fixada no solo, de maneira que esta ficou entre os pés do avaliado, facilitando desta forma a visualização do avaliador do local de aterrizagem. A execução do teste teve a seguinte técnica: o aluno em pé atrás da linha inicial saltou a maior distância possível à frente, com a ajuda da flexão das pernas e utilizando o balanço dos braços. $O$ resultado foi conhecido pela distância entre a linha inicial e o calcanhar mais próximo da linha inicial(14).

Para velocidade usou-se a corrida de 20 metros de saída parada. Para execução, o avaliado saiu na posição em pé com uma das pernas na linha pré-determinada, demarcada com três linhas paralelas no solo da seguinte forma: a primeira (linha de partida); a segunda, distante 20 metros da primeira (linha de chegada) e a terceira linha (linha de referência), marcada a dois metros da segunda (linha de chegada). Deslocaram-se o mais rápido possível até cruzar a linha de 20 metros, sendo que o cronômetro foi acionado no momento em que o avaliado realizou o primeiro passo e travado no exato momento que estes cruzavam a linha de chegada (4).

Para o teste de agilidade, usou-se a corrida sinuosa. $\mathrm{O}$ avaliado realizou um percurso sinuoso, demarcado por cinco cones, distantes 1,50 metros entre si, estando o primeiro distante a 3 metros da linha de partida, num total de 9 metros. $O$ aluno realizou o percurso correndo entre os cones nos sentidos de ida e volta o mais rápido possível. O cronômetro foi acionado no momento em que o avaliado realizou o primeiro passo e travado no exato momento que estes cruzavam a linha de chegada(15).

$\mathrm{O}$ teste de agilidade foi medido através da corrida 
alternada de 9,14 metros. Como material foram utilizados 2 blocos de madeira $(5 \mathrm{~cm} \times 5 \mathrm{~cm} \times 10 \mathrm{~cm})$ e um cronômetro. Foram traçadas duas linhas paralelas ao solo em uma distância de 9,14 metros marcados pela borda externa, onde foram colocados os dois blocos de madeira, a aproximadamente $10 \mathrm{~cm}$ da linha externa, deixando $30 \mathrm{~cm}$ de espaço entre os dois. Após um sinal sonoro, realizado pelo avaliador, os alunos deveriam correr em direção aos blocos, pegando um deles e voltando para a linha inicial, colocando-o atrás da linha. Em seguida, os alunos, deveriam correr de volta para pegar o segundo bloco, carregando-o até a linha de partida. Orientouse aos executantes para não jogarem os blocos e sim colocá-los junto ao solo. O teste somente foi terminado e o cronômetro parado quando o avaliado colocou o último bloco no solo e ultrapassou, com pelo menos um dos pés, a linha que delimitava os espaços demarcados ${ }^{(14)}$.

Corrida sinuosa com bola - Da mesma forma que a corrida sinuosa sem bola, o avaliado percorreu um percurso sinuoso, demarcado por cinco cones, distante 1,50 metros entre si, estando o primeiro distante a 3 metros da linha de partida, totalizando 9 metros. $O$ aluno realizou o percurso correndo entre os cones nos sentidos de ida e volta, o mais rápido possível, porém conduzindo uma bola com os pés. O cronômetro foi acionado no momento em que o testando realizou o primeiro passo e travado no exato momento que estes cruzavam a linha de chegada, adaptado de Pitanga ${ }^{(15)}$.

Para avaliação dos testes de precisão dos membros inferiores foram realizados chute a gol com bola parada e chute a gol com bola em movimento e o passe. Para o teste de chute a gol com a bola parada, o avaliado posicionou-se em pé parado a uma distância de 15 metros do gol da quadra de futsal e chutou a bola com o pé da perna predominante, tentando marcar o gol. Foram realizadas 10 tentativas sem tempo pré-determinado. $O$ avaliador anotou a quantidade de gols realizados.

Para avaliação da precisão de chute a gol com a bola em movimento, o avaliado posicionou-se em pé e parado a uma distância de 10 metros do gol da quadra de futsal e o avaliador posicionou-se em uma das linhas de fundo onde realizava um passe para o avaliado, que deveria chutar a bola a gol sem dominar a mesma, ou seja, "chutar de primeira". Foram anotados somente os gols realizados, adaptado de Gomes e Machado(4).

Para avaliação do passe, foi demarcada uma distância de 10 metros entre o avaliador e o avaliado, sendo que o avaliador poderia movimentar-se numa área de $60 \mathrm{~cm}$ para receber o passe do avaliado. O avaliado deveria realizar 10 passes validando somente os passes certos, adaptado de Gomes e Machado(4).

A análise dos dados foi feita por meio de uma estatística descritiva, (média e desvio padrão). Foi verificada a normalidade dos dados (KolmogorovSmirnov) e aplicado ANOVA para medidas repetidas, com post hoc de Bonferroni. Foram utilizados os pacotes estatísticos SPSS (12.0) e o Instat (3.0). Foi adotado o nível de significância $p<0,05$.

\section{RESULTADOS}

Os dados apresentaram distribuição normal. Os resultados do presente estudo estão expressos na Tabela 1, através de Media e desvio padrão, sendo que não houve diferença estatisticamente significativa entre os momentos (pré e pós), bem como entre os grupos FC e FA.

\begin{tabular}{|c|c|c|c|c|}
\hline \multicolumn{5}{|c|}{ TABELA 1: RESULTADOS PRINCIPAIS } \\
\hline \multirow[t]{2}{*}{ TESTES } & & & & \multirow{2}{*}{$\begin{array}{l}\text { Pós } \\
\text { M } \\
\text { dp }\end{array}$} \\
\hline & $M^{\text {Pós }} \mathrm{dp}$ & $M^{\text {Pós }} \mathrm{dp}$ & $M^{\text {Pós }} \mathrm{dp}$ & \\
\hline $\begin{array}{l}\text { Salto } \\
\text { horizontal }\end{array}$ & $1,33 \pm 0,2$ & $1,43 \pm 0,2$ & $1,27 \pm 0,2$ & $\begin{array}{l}1,36 \pm \\
0,2\end{array}$ \\
\hline $\begin{array}{l}\text { Corrida vai e } \\
\text { vem }\end{array}$ & $\begin{array}{l}12,25 \pm \\
2,8\end{array}$ & $\begin{array}{l}11,89 \pm \\
0,9\end{array}$ & $\begin{array}{l}12,54 \pm \\
1,1\end{array}$ & $\begin{array}{l}12,36 \\
\pm 0,9\end{array}$ \\
\hline $\begin{array}{l}\text { Corrida } \\
\text { Sinuosa }\end{array}$ & $8,15 \pm 0,8$ & $7,69 \pm 0,7$ & $8,04 \pm 0,8$ & $\begin{array}{l}7,86 \pm \\
1,9\end{array}$ \\
\hline Abdominal & $\begin{array}{l}31,32 \pm \\
8,6\end{array}$ & $\begin{array}{l}32,84 \pm \\
7,3\end{array}$ & $\begin{array}{l}32,7 \pm \\
10,2\end{array}$ & $\begin{array}{l}32,3 \pm \\
10,4\end{array}$ \\
\hline $\begin{array}{l}\text { Corrida } \\
\text { sinuosa com } \\
\text { bola }\end{array}$ & $\begin{array}{l}20,62 \pm \\
6,9\end{array}$ & $\begin{array}{l}20,15 \pm \\
6,4\end{array}$ & $19,2 \pm 7,3$ & $\begin{array}{l}17,99 \\
\pm 8,1\end{array}$ \\
\hline Chute a gol & $4,16 \pm 2,1$ & $5,89 \pm 2,1$ & $5,45 \pm 1,9$ & $\begin{array}{l}6,9 \\
\pm 2\end{array}$ \\
\hline $\begin{array}{l}\text { Chute a gol } \\
\text { bola em } \\
\text { movimento }\end{array}$ & $4,11 \pm 2$ & $5,42 \pm 2$ & $5,25 \pm 1,5$ & $\begin{array}{l}6,05 \\
\pm 2\end{array}$ \\
\hline Passe & $3,68 \pm 2,1$ & $4,68 \pm 1,9$ & $4,2 \pm 1,8$ & $\begin{array}{l}6,25 \pm \\
1,4\end{array}$ \\
\hline Corrida $20 \mathrm{~m}$ & $4,61 \pm 0,4$ & $4,65 \pm 0,4$ & $4,6 \pm 0,6$ & $\begin{array}{l}4,43 \pm \\
0,6\end{array}$ \\
\hline
\end{tabular}


Valores médios (M) e desvio padrão (dp) para os grupos futsal e capoeira $(F C)$ e futsal e atletismo (FA) para os testes salto horizontal $(\mathrm{m})$, corrida vai e vem (seg), corrida sinuosa (seg), abdominal (rpts), corrida sinuosa com bola (seg), chute a gol (rpts), chute a gol bola em movimento (rpts), passe (rpts), corrida de 20m (seg).

\section{DISCUSSÃO}

O presente estudo se propôs a avaliar a influência do treinamento concorrente envolvendo atletismo (A) e capoeira (C) em crianças de 7 a 10 anos praticantes de futsal $(F)$ e constatamos que não houve diferença significante estatisticamente entre os grupos e pré e pós dentro dos grupos, nos testes motores investigados. Porém, os grupos apresentaram uma tendência no ganho de suas habilidades motoras.

Para Kunze apud Passos( ${ }^{(16)}$, os exercícios que exigem uma inversão rápida dos movimentos, com participação de todo corpo, ajudam no desenvolvimento da agilidade. Estes achados corroboram com os estudos de Nacif(17), que afirma que a capoeira, como outras modalidades esportivas, propicia o desenvolvimento da coordenação motora, e se inserida como conteúdo das aulas de Educação Física, se constitui em ferramenta eficaz no desenvolvimento das habilidades motoras dos escolares.

Martins ${ }^{(18)}$ demonstrou a contribuição da capoeira no desenvolvimento psicomotor de crianças entre seis e dez anos de idade e conclui que a vivência corporal existente na capoeira auxilia no desenvolvimento de todos os aspectos psicomotores, na aquisição, estabilização e diversificação de habilidades básicas e posteriormente a aprendizagem de tarefas mais complexas.

O treinamento esportivo é definido por um processo de ações complexas, planejadas e orientadas que visam o melhor desempenho esportivo possíve|(19).

O melhor método de treinamento é aquele que a criança aprenda de maneira rápida, segura e com prazer ${ }^{(20)}$. A criança vem em primeiro lugar e o método deve se unir a habilidade que a criança tem de criar, interagir e improvisar. Desta forma ela vivenciará novas experiências $^{(3)}$. No jogo, são apresentadas diversas situações que devem ser resolvidas em todos os momentos e de maneiras diferentes, aumentando assim o seu repertório motor(21). Recomenda-se a reprodução do presente estudo, porém, com um espaço de tempo maior entre o pré e pós-teste.

A literatura científica quanto ao treinamento concorrente envolvendo diferentes práticas esportivas tem sido pouco estudada.

\section{CONCLUSÃO}

As crianças de 7 a 10 anos praticantes de futsal não obtiveram ganhos de desenvolvimento motor com a prática de treinamento concorrente, durante 2 meses, envolvendo tanto capoeira quanto atletismo. Porém apresentaram uma tendência a melhorarem.

Valores médios (M) e desvio padrão (dp) para os grupos futsal e capoeira (FC) e futsal e atletismo (FA) para os testes salto horizontal $(\mathrm{m})$, corrida vai e vem (seg), corrida sinuosa (seg), abdominal (rpts), corrida sinuosa com bola (seg), chute a gol (rpts), chute a gol bola em movimento (rpts), passe (rpts), corrida de $20 \mathrm{~m}$ (seg).

\section{REFERÊNCIAS BIBLIOGRÁFICAS}

1. Mutti D. Futsal da iniciação ao alto nível. São Paulo: Loyola, 1999.

2. Costa CF. Futsal aprenda ensinar. Florianópolis: VisualBooks, 2003.

3. Santana WC de. Futsal: apontamentos pedagógicos na iniciação e na especialização. São Paulo: Autores associados, 2004.

4. Gomes AC, Machado JA. Futsal metodologia e planejamento na infância e adolescência. Londrina: Midiograf, 2001.

5. Magill RA. Motor learning: concepts and applications. $4^{\text {a }}$ ed. São Paulo: Edgard Blücher, 2000.

6. Weineck J. Anatomia aplicada ao esporte. $3^{\mathrm{a}}$ ed. São Paulo: Manole, 1986.

7. Oliveira MC. Atletismo escolar: uma proposta de ensino na Educação infantil. Rio de Janeiro: Sprint, 2006.

8. Campos H. Capoeira na escola. Salvador: Presscolor, 1990.

9. Brasil - Secretaria de educação fundamental Parâmetros Curriculares Nacionais (PCNs): Educação Física / Brasília: MEC / SEF, 1998.

10. Barbanti VJ. Teoria e prática do treinamento desportivo. $2^{\mathrm{a}}$ ed. São Paulo: Edgard Brucher, 1997. 11. Panissa VLG. Exercício Concorrente. Analise do efeito agudo da ordem de execução sobre o gasto energético total: Rev Bras Med Esporte 2009;15(2):127131.

12. Hakkinen K, Alen M, Kraemer WJ, Gorostiaga $\mathrm{E}$, Izquierdo $\mathrm{M}$, rusko $\mathrm{H}$, et al. Neuromuscular 
adaptations during concurrent strength and endurance training versus strength training. Eur J Appl Physiol 2003;89(1):42-52.

13. Paulo AC. Efeito do treinamento concorrente no desenvolvimento da força motora e da resistência aeróbia. Revista Mackenzie de Educação Física e Esporte - 2005;4(4):145-154.

14. Johnson BL, Nelson JK. Practical measurements for evaluation in physical education. Minnesota: Burgess Publishing Company, 1979.

15. Pitanga FJG. Testes, medidas e avaliação física e esportes. $5^{\mathrm{a}}$ ed. São Paulo: Phorte, 2008.

16. Passos WS. A influência do treinamento de futsal na velocidade e agilidade de escolares, EF Deportes 2009;13(129).

17. Nacif $C$, Moraes JM. Comparação da influência da capoeira na coordenação motora de escolares, EF Deportes 2010;14(137).

18. Martins SF. A contribuição da capoeira no desenvolvimento psicomotor de crianças entre seis e dez anos de idade. Apresentação de monografia à Universidade Candido Mendes como condição prévia para conclusão do curso de pós-graduação, rio de Janeiro, 2004.

19. Weineck J. Treinamento ideal. 9a ed. São Paulo: Manole, 1999.

20. Venlioles FM. Escola de futebol. $2^{\mathrm{a}}$ ed. Rio de Janeiro: Sprint, 2004.

21. Melo RS. Jogos recreativos para futebol. $2^{\mathrm{a}}$ ed. Rio de Janeiro: Sprint, 2002.

Endereço para correspondência:

UCB - LAFIT - QS 07, Lote 01, S/N, Bloco G, Sala 120 D. - CEP:

71966-700.

Bairro Areal. Taguatinga-DF - Telefone: (61) 3356-9044. - Fax: (61)

3356-9350.

e-mail: bibiano.madrid@gmail.com 